\title{
Basic Physics for a Surgeon
}

\author{
Sukriti Rastogi ${ }^{1} \cdot$ Varidh Katiyar $^{2}$ (D)
}

Received: 21 July 2018 / Accepted: 23 July 2018 / Published online: 27 July 2018

(C) Association of Surgeons of India 2018

Dear editor,

We read with keen interest the article regarding the physical principles important for a surgeon titled "Rajkumar, J., Chopra, P., and Chintamani. (2015). Basic Physics Revisited for a Surgeon." The Indian Journal of Surgery, 77(3), 169-175 [1].

We would like to commend the authors for highlighting the importance of knowledge of basic principles of physics for surgeons, an inspiration that drives all the major breakthroughs in scientific disciplines. However, we would also like to point out certain issues, e.g., gastro-esophageal reflux disease (GERD) and the role of Poiseulli's theorem. The compression of lower esophagus by raised intra-abdominal pressure post prandially plays a major role in preventing reflux. The physics behind compressibility of compliant tubular structures under external pressure explained by soft straw experiment has been wrongly attributed to Poiseulli's equation. For this, the authors must have explained how the pressures inside a fluid increase in direct proportion to the depth. Even this fails to capture what actually happens in this situation as the compression of esophagus due to intra-abdominal pressure is not akin to greater compression at increasing depth in a fluid.

Another example of Poiseulli's equation is for correcting arterial narrowing at two or more sites. We would like to explain this concept further using hypothetical values as it might initially seem counter intuitive to some of the intended

Varidh Katiyar

katiyar.varidh@gmail.com

Sukriti Rastogi

sukriti.rastogi06@gmail.com

1 Department of Surgery, Dr. Ram Manohar Lohia Hospital and Post Graduate Institute of Medical Education and Research, New Delhi, India

2 Department of Neurosurgery, All India Institute of Medical Sciences, Room No 7/96, Gents Hostel, New Delhi 110029, India readers that how a similar increase in radius may result in greater increase in the flow rate in larger artery though the relative increase in radius is lesser for the larger artery. Let us suppose there are two arteries with radii of 3 units and 4 units, then the flow rate should be $3^{\wedge} 4=81$ and $4^{\wedge} 4=$ 256 units. On increasing the radii of both by 1 unit, the flow rates become 256 units and $5^{\wedge} 4=625$ units. An increase of 175 units in the smaller and 369 units in the larger artery though there was $33 \%$ relative increase in radius of smaller artery compared to only $25 \%$ in the larger artery.

Bernoulli's principle is primarily about the radial pressure at the site of high-velocity flow rather than proximal rise in pressure due to increased resistance distally. In nonrigid tubular structures, it does not explain in entirety the concept of treatment of leak after sleeve gastrectomy with stent placement.

The authors also seemed to have simplified the principles of diathermy and have not explained the role of the different frequency and duration of stimulation in addition to current density in deciding the relative amounts of cutting and coagulation of the tissues.

Concluding, we agree with the authors on the importance of understanding basic principles of physics. We have highlighted certain aspects that the authors have either missed or might have omitted due to the intended readership belonging to a biological science domain.

\section{Compliance with Ethical Standards}

Conflict of Interest The authors declare that they have no conflict of interest.

\section{Reference}

1. Rajkumar J, Chopra P, Chintamani (2015) Basic physics revisited for a surgeon. The Indian Journal of Surgery 77(3):169-175. https://doi.org/10.1007/s12262-015-1308-6 